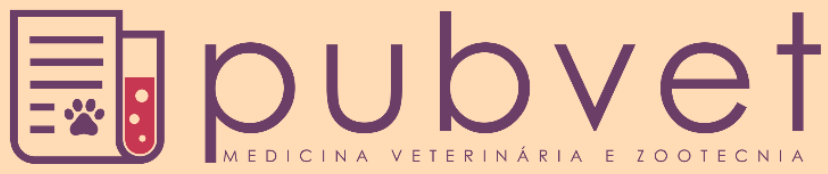

https://doi.org/10.31533/pubvet.v16n01a1010.1-7

\section{Estudo do comportamento de microrganismos psicrotróficos proteolíticos isolados de leite cru refrigerado}

\author{
Juliana Querino Goulart ${ }^{1 \oplus,}$, Poliana Cristiane do Prado ${ }^{2 \oplus}$, Carolina Laipelt Matias ${ }^{2 \oplus}$, Andrea \\ Troller Pinto ${ }^{3 *}$
}

${ }^{1}$ Doutoranda do Programa de Pós-graduação em Agronegócios, Universidade Federal do Rio Grande do Sul. Porto Alegre - RS. ${ }^{2}$ Graduanda em Medicina Veterinária, Universidade Federal do Rio Grande do Sul. Porto Alegre - RS.

${ }^{3}$ Professora da Universidade Federal do Rio Grande do Sul. Porto Alegre - RS.

*Autor para correspondência, E-mail: andrea.troller@ufrgs.br

Resumo. Temperaturas de resfriamento do leite facilitam a multiplicação de microrganismos psicrotróficos que produzem enzimas termorresistentes com potencial para degradar os componentes do leite. Visando avaliar o comportamento de microrganismos psicrotróficos em leite cru refrigerado, este estudo teve por objetivo verificar a multiplicação e atividade enzimática de leites contaminados intencionalmente com tais microrganismos em temperatura de refrigeração. Também teve a intenção de avaliar a atividade enzimática durante a pasteurização lenta (LTBT). Para isso, microrganismos psicrotróficos produtores de enzimas proteolíticas foram isolados de leite cru refrigerado. Esses microrganismos foram testados quanto ao seu tempo de geração em temperatura de $7 \pm 1^{\circ} \mathrm{C}$ por oito dias em dois diferentes meios: Caldo cérebro coração (BHI) e leite desnatado reconstituído a 10\% (LDR). Também foram avaliados quanto à produção de proteases e suas respectivas atividades enzimáticas durante a pasteurização lenta. Os resultados demonstraram que os isolados não atingiram a fase estacionária na curva de crescimento bacteriano durante os oito dias de incubação. Além disso, atividades das enzimas proteolíticas foram detectadas mesmo após o tratamento térmico, indicando resistência das mesmas às temperaturas da pasteurização lenta. Concluindo, para evitar perdas econômicas associadas à atividade enzimática, sugere-se que haja maior controle sanitário do ambiente de produção, assim como reduzidos períodos de armazenamento anteriores ao processamento térmico, visando reduzir a multiplicação de psicrotróficos proteolíticos.

Palavras-chave: Atividade enzimática, curvas de crescimento, pasteurização lenta

\section{Behavior's study of psychrotrophic microorganisms isolated from cooled raw milk}

Abstract. Milk cooling temperatures facilitate the multiplication of psychrotrophic bacteria that produce thermoresistant enzymes with potential to degrade milk's components. Aiming to evaluate the behavior of psychrotrophs in cooling raw milk, this study aimed to verify the multiplication and enzymatic activity of milk intentionally contaminated with such microorganisms at refrigeration temperature. Also, it was intended to evaluate the enzymatic activity during long pasteurization (LTST). For this, proteolytic psychrotrophs were isolated from refrigerated raw milk. These microorganisms were tested for their generation time at a temperature of $7 \pm 1^{\circ} \mathrm{C}$ for 192 hours in two different media: Brain Heart Infusion broth (BHI) and 10\% reconstituted skim milk (LCR). They were also evaluated for the production of proteases and their respective enzymatic activities during slow pasteurization. The results demonstrated that the isolates did not reach the stationary 
phase in the bacterial growth curve during the eight days of incubation. Furthermore, proteolytic enzyme activities were detected even after heat treatment, indicating resistance to slow pasteurization temperatures. In conclusion, to avoid economic losses associated with enzymatic activity, it is suggested that there is greater sanitary control of the production environment, as well as reduced storage periods prior to thermal processing, in order to reduce the multiplication of proteolytic psychrotrophs.

Keywords: enzymatic activity, growth curves, long pasteurization

\section{Introdução}

A refrigeração é uma das soluções mais eficazes na redução das perdas econômicas devido à deterioração provocada por microrganismos mesófilos, que se multiplicam rapidamente no leite armazenado em temperatura ambiente. No entanto, as baixas temperaturas facilitam a multiplicação de microrganismos psicrotróficos, que se tornaram prevalentes na microbiota do leite cru refrigerado. Estes microrganismos são conhecidos por produzir enzimas líticas que degradam principalmente proteínas e gorduras (Vithanage et al., 2016; Zhang et al., 2020). Os tratamentos térmicos convencionais aos quais é submetido o leite para consumo humano, elimina grande parte dos microrganismos psicrotróficos, porém as enzimas podem resistir à temperatura elevada, estando ativas após a morte do microrganismo que as produziram (Baur et al., 2015; Marchand et al., 2017; Stoeckel et al., 2016a; Stoeckel et al., 2016b).

As perdas econômicas relacionadas às proteases de origem microbiana estão ligadas à redução do rendimento e alteração das características intrínsecas dos derivados lácteos. Por isso, medidas preventivas como controle sanitário dos ambientes da produção, assim como a redução no tempo de armazenamento do leite antes do processamento são desejáveis à melhoria da qualidade deste produto. Menores contaminações iniciais por microrganismos psicrotróficos (obtidos com o controle higiênico do ambiente), assim como tratamento térmico realizado o mais rápido possível (evitando a multiplicação destes microrganismos) são úteis na redução da produção de enzimas, já que este processo se inicia quando as contagens de microrganismos psicrotróficos atingem valores superiores a seis ciclos logarítmicos (Muir \& Kjaerbye, 1996; Nörnberg et al., 2009; Reche et al., 2015; Zhang et al., 2020).

Visando avaliar o comportamento de microrganismos psicrotróficos em leite cru refrigerado, este estudo teve por objetivo verificar a multiplicação e atividade enzimática de leites contaminados intencionalmente com tais microrganismos em temperatura de refrigeração. Além disso, teve a intenção de avaliar a atividade enzimática durante a pasteurização lenta.

\section{Material e métodos}

Amostras de leite cru refrigerado foram coletadas em triplicata em duas diferentes oportunidades, diretamente de tanque de resfriamento de uma unidade produtora de leite. As amostras foram armazenadas em frascos estéreis, acondicionadas em temperaturas inferiores a $10^{\circ} \mathrm{C}$ e encaminhadas ao Laboratório de Inspeção e Tecnologia de Leites e Derivados, Ovos e Mel da Universidade Federal do Rio Grande do Sul.

No laboratório, diluições decimais foram inoculadas em Ágar de Contagem Padrão (PCA) e mantidas a $36 \pm 1^{\circ} \mathrm{C}$ por $48 \mathrm{~h}$ para contagem de microrganismos mesófilos, enquanto a contagem de psicrotróficos foi realizada após armazenamento por 10 dias a $7 \pm 1^{\circ} \mathrm{C}$ (Mörschbächer et al., 2018). Com base nas diferentes morfologias das colônias observadas durante a contagem, os microrganismos foram isolados e mantidos em cultura pura.

Os microrganismos mesófilos foram testados quanto a sua capacidade de se multiplicar em temperaturas de refrigeração, sendo inoculados em Ágar Triptona de Soja (TSA) e armazenados em temperatura de $7^{\circ} \pm 1^{\circ} \mathrm{C}$ durante 10 dias. A capacidade de produzir enzimas proteolíticas dos isolados foi avaliada conforme BRASIL (1993). Para verificar a qualidade do meio teste foi utilizada como controle positivo a Pseudomonas aeruginosa (ATCC 27853), uma cepa sabidamente proteolítica. A identificação dos microrganismos psicrotróficos proteolíticos foi realizada por espectrometria de massa com ionização de dessorção a laser e análise de tempo de voo (MALDI TOF MS, AutoflexSpeed ${ }^{\circledR}$, programas FlexControl ${ }^{\circledR}$ e Biotyper ${ }^{\circledR}$-Bruker, Alemanha), usando o método de extração do ácido fórmico com etanol (Almuzara et al., 2015; Jang \& Kim, 2018). 


\section{Curvas de crescimento}

Os microrganismos psicrotróficos proteolíticos foram testados quanto ao seu tempo de geração na temperatura de $7 \pm 1^{\circ} \mathrm{C}$ em dois diferentes meios: Caldo infusão de cérebro e coração (BHI) e em meio leite desnatado reconstituído a 10\% (LDR). As contagens foram feitas às 12, 24, 48, 72, 96, 120, 144, 168 e 192 horas após a inoculação, realizando diluições decimais sucessivas inoculadas em PCA e incubadas $7 \pm 1 \mathrm{C}$ por 10 dias. Todas as análises foram feitas em triplicata.

\section{Atividade enzimática durante a pasteurização lenta}

Cada um dos microrganismos psicrotróficos proteolíticos isolados foi cultivado em BHI por sete dias a $7 \pm 1^{\circ} \mathrm{C}$ para obtenção de concentração de $10^{9} \mathrm{UFC} / \mathrm{mL}$ (unidades formadoras de colônias/mililitro). Para confirmar essa concentração foi utilizada a escala nefelométrica de Mc Farland, selecionando o tubo cinco como comparação, obtendo-se meios que apresentavam concentração próxima a 1,5 bilhões de microrganismos. Um mililitro deste conteúdo foi transferido para um frasco contendo $99 \mathrm{~mL}$ de LDR, resultando em uma contagem de $10^{6} \mathrm{UFC} / \mathrm{mL}$ e acondicionado a $7 \pm 1^{\circ} \mathrm{C}$ por 72 horas.

Para testar a termo resistência das proteases produzidas pelos microrganismos psicrotróficos proteolíticos isolados, os frascos foram retirados da refrigeração e submetidos à pasteurização lenta, sendo coletadas amostras para mensuração da atividade enzimática à medida que o leite era aquecido. Foram quantificadas as atividades enzimáticas nas temperaturas de $10^{\circ}, 20^{\circ}, 30^{\circ}, 40^{\circ}, 50^{\circ}, 60^{\circ}, 65^{\circ} \mathrm{C}$ e durante os 10,20 e 30 min de manutenção na temperatura de $65^{\circ} \mathrm{C}$. A determinação da atividade foi realizada pelo método de hidrólise da gelatina solúvel descrito por Nörnberg et al. (2009).

\section{Resultados e discussão}

As médias dos resultados das contagens de microrganismos mesófilos e psicrotróficos podem ser visualizadas na tabela 1.

Tabela 1. Médias das contagens de microrganismos mesófilos e psicrotróficos das amostras de leite cru refrigerado.

\begin{tabular}{lcc}
\hline Coleta & 1 & 2 \\
\hline Mesófilos totais & $5,23 \pm 0,27$ & $6,45 \pm 0,32$ \\
Psicrotróficos totais & $5,29 \pm 0,29$ & $5,29 \pm 0,17$ \\
\hline
\end{tabular}

Os valores médios das contagens de mesófilos e psicrotróficos são semelhantes aos obtidos em estudos anteriores realizados no Rio Grande do Sul. Porém, cabe ressaltar que é possível grande variação nestes números, já que as contagens de microrganismos estão associadas a fatores que variam de acordo com a unidade produtora, sendo eles a região onde o leite é produzido, o tipo de ordenha, o estado sanitário do rebanho, o manejo dos animais, a higienização de equipamentos utilizados na ordenha e armazenamento do leite, entre outros (Arcuri et al., 2008; Borges et al., 2009; Reche et al., 2015; Silveira \& Bertagnolli, 2014; Vargas et al., 2014).

Ao todo, foram isolados nove microrganismos psicrotróficos. Desses, apenas três apresentaram capacidade de produzir proteases e foram identificados como pertencentes aos gêneros Buttiauxella, Alcaligenes e Pseudomonas. Buttiauxella spp. e Alcaligenes têm sido identificados em leite cru refrigerado e derivados lácteos, porém normalmente não estão associados a produção de proteases. Entretanto, Pseudomonas é um gênero comumente associado à produção de enzimas líticas, sendo frequentemente isolado em leite cru refrigerado devido à sua capacidade de se multiplicar em temperaturas de refrigeração e de produzir biofilmes nos equipamentos de ordenha e armazenamento do leite (Moonga et al., 2020; Teh et al., 2014; Vithanage et al., 2016; von Neubeck et al., 2015; Zhang et al., 2020).

\section{Curvas de crescimento}

Os resultados da multiplicação dos microrganismos em BHI e LDR são apresentados nas figuras 1, $\underline{2}$ e $\underline{3}$. Foi observado que os isolados tiveram uma fase inicial com menores taxas de crescimento, o que perdurou por aproximadamente 12 horas. Nas 180 horas seguintes os microrganismos se multiplicaram lentamente, não atingindo a fase estacionária bem definida no período avaliado. As taxas de crescimento médio (TCM) foram calculadas pela diferença entre o número de microrganismos inicial e final divididos pelo número de horas contabilizadas, sendo o resultado expresso em UFClog 10/h. A TCM para os microrganismos isolados são - em LDR e BHI - respectivamente: Buttiauxella sp. 0,052 e 0,047 
UFClog 10/h; Alcaligenes sp. 0,056 e 0,054 UFClog10/h; Pseudomonas sp. 0,046 e 0,047 UFClog10/h. Pode-se observar que a TCM da Buttiauxella sp. foi maior no leite, porém, ao visualizar a curva de crescimento, é observado um declínio no número de microrganismos no BHI nas últimas $24 \mathrm{~h}$, o que afetou diretamente no cálculo desta taxa. Alcaligenes sp. teve comportamento semelhante nos dois meios avaliados, com exceção do período entre as 12 e 36h, onde houve maior crescimento no BHI. A TCM deste microrganismo foi superior no LD. Já a Pseudomonas sp. obteve maior TCM em BHI, apresentando pequenas diferenças entre as curvas obtidas nos dois diferentes meios.

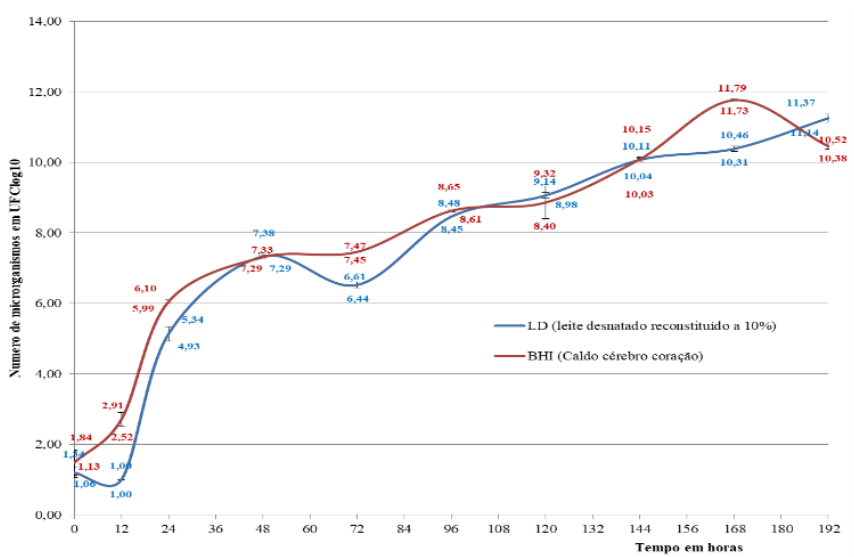

Figura 1. Curva de crescimento de Buttiauxella sp. em BHI e LD na temperatura de $7 \pm 1^{\circ} \mathrm{C}$. $*$ Os números apresentados na figura acima, que possuem a mesma cor da legenda, referem-se aos valores médios adicionados ou subtraídos dos desvios padrões calculados.

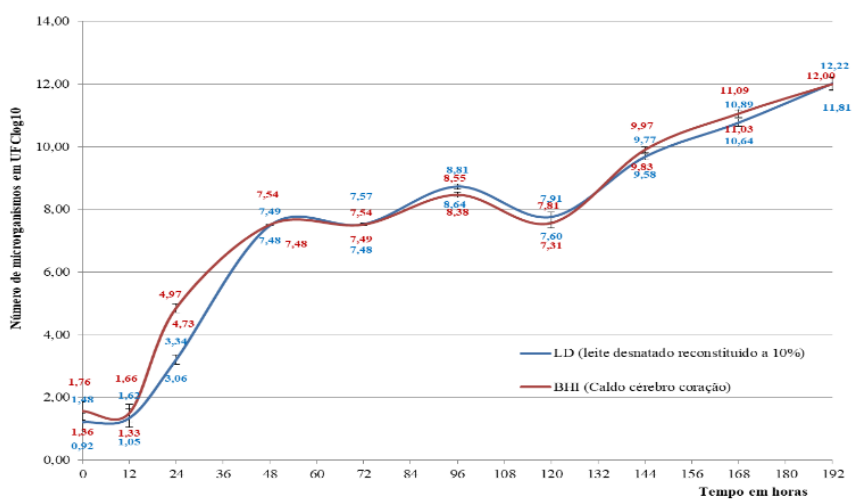

Figura 2. Curva de crescimento de Alcaligenes sp. em BHI e LD na temperatura de $7 \pm 1^{\circ} \mathrm{C}$. *Os números apresentados na figura acima, que possuem a mesma cor da legenda, referem-se aos valores médios adicionados ou subtraídos dos desvios padrões calculados.

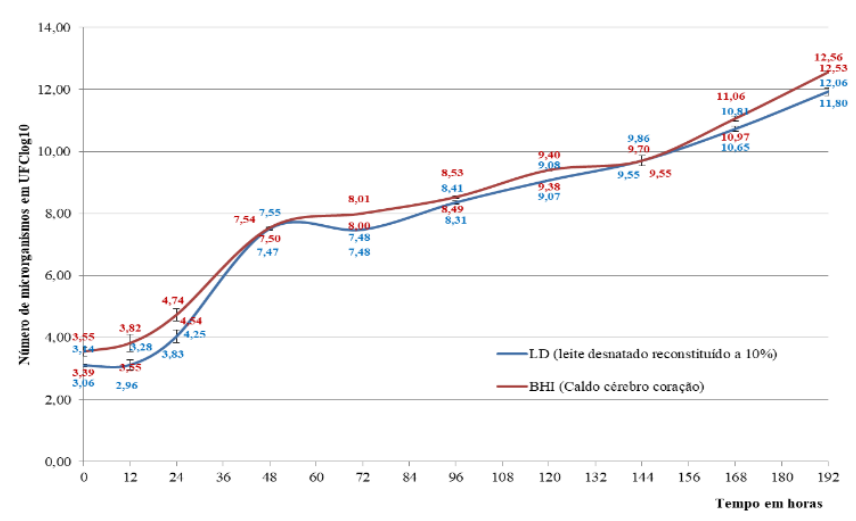

Figura 3. Curva de crescimento de Pseudomonas sp. em BHI e LD na temperatura de $7 \pm 1^{\circ} \mathrm{C}$. $*$ Os números apresentados na figura acima, que possuem a mesma cor da legenda, referem-se aos valores médios adicionados ou subtraídos dos desvios padrões calculados. 
Estudos realizados por Stoeckel et al. (2016a) e Stoeckel et al. (2016b) avaliaram as fases do crescimento a $6^{\circ} \mathrm{C}$ de cepas de espécies do gênero Pseudomonas sp. e observaram que elas atingiam a fase estacionária dentro de 3-4 dias. O mesmo não ocorreu no presente estudo, já que não se observou a fase estacionária do crescimento bacteriano após oito dias a $7 \pm 1^{\circ} \mathrm{C}$.

Microrganismos psicrotróficos quando submetidos a temperaturas próximas a $7 \pm 1 \mathrm{C}$ apresentam fases de adaptação mais longas quando comparadas a dos mesófilos em temperaturas superiores a $30 \pm$ $1 \mathrm{C}$. Isso foi determinante na definição dos tempos de refrigeração máximos permitidos pela legislação, onde são permitidas $48 \mathrm{~h}$ de armazenamento do leite cru na propriedade rural em temperaturas iguais ou inferiores a $4^{\circ} \mathrm{C}$ (Baur et al., 2015; BRASIL, 2018; Marchand et al., 2017; Stoeckel et al., 2016a; Stoeckel et al., 2016b; Xin et al., 2017; Yuan et al., 2019).

\section{Atividade enzimática durante a pasteurização lenta}

Os resultados da atividade enzimática do LDR contaminado com Pseudomonas sp., Alcaligenes sp e Buttiauxella sp. durante a pasteurização lenta é apresentado na figura 4.

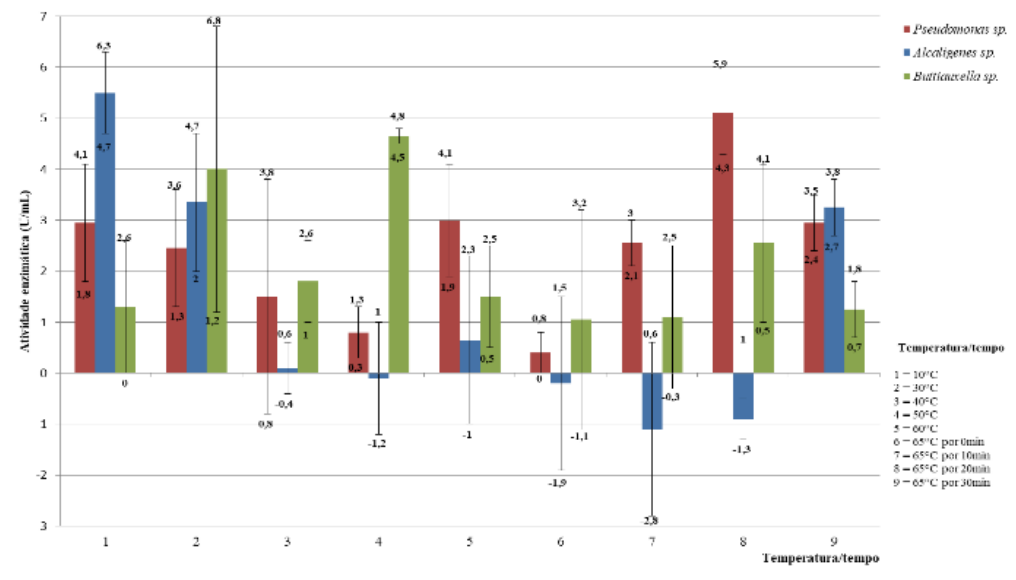

Figura 4. Atividade enzimática $(\mathrm{U} / \mathrm{mL})$ do leite contaminado com Pseudomonas sp., Alcaligenes sp. e Buttiauxella sp durante a pasteurização lenta.

Antes de iniciar o aquecimento (temperatura de $10^{\circ} \mathrm{C}$ ) o LDR contaminado com Alcaligenes sp. apresentava a maior atividade enzimática. À medida que ia aquecendo ela diminuiu, porém houve aumento na última aferição (aos $65^{\circ} \mathrm{C}$ por $30 \mathrm{~min}$ ). Esse comportamento errático também foi observado no LDR contaminado com Pseudomonas sp. e Buttiauxella sp., havendo aumentos e diminuições durante a pasteurização lenta. Entretanto, ao final do tratamento térmico, todos os LDR apresentaram atividades enzimáticas residuais, ou seja, as enzimas proteolíticas não foram completamente inativadas pelo aquecimento.

Estudos anteriores também identificaram atividades de enzimas proteolíticas após os tratamentos térmicos, sendo a pasteurização e o tratamento UHT não são suficientes para inativar tais enzimas. Estudo realizado com proteases produzidas por cepas de Pseudomonas sp., determinou que algumas delas resistiam a temperaturas superiores a $100^{\circ} \mathrm{C}$ (Marchand et al., 2009; Pinto et al., 2015; $\underline{\text { Stoeckel }}$ et al., 2016a; Stoeckel et al., 2016b).

A medida de atividade proteolítica pelo método de gelatina solúvel indica presença de proteases, porém sua origem não é necessariamente microbiana. O leite contém uma grande variedade de enzimas que podem ter origem na própria glândula mamária ou nas células sanguíneas. Enzimas próprias do leite, como a plasmina, podem ter sua atividade aumentada durante o tratamento térmico, já que ocorre a inativação térmica dos inibidores da conversão de plasminogênio (zimogênio) em plasmina aumentando os níveis dela no leite. No entanto, a plasmina não está associada à degradação da caseína como as proteases de origem microbiana, cujas atividades alteram os sítios da principal molécula proteica láctea, afetando o rendimento e a qualidade dos seus derivados (Ismail \& Nielsen, 2010; Marchand et al., 2017; Stoeckel et al., 2016; Stoeckel et al., 2016).

Devido ao efeito das proteases microbianas no leite e seus derivados, a redução da contaminação por microrganismos psicrotróficos produtores destas enzimas é essencial à qualidade desses produtos. 
Zhang et al. (2020) demonstraram que leites armazenados em temperatura de $7^{\circ} \mathrm{C}$ por cinco dias atingem contagens superiores a $7 \log 10 \mathrm{UFC} / \mathrm{ml}$ de microrganismos psicrotróficos o que, segundo Muir \& Kjaerbye (1996) é mais que suficiente para produção de enzimas, sendo que esta se inicia em contagens acima de $6 \log 10 \mathrm{UFC} / \mathrm{mL}$. Portanto, a adoção de medidas higiênico-sanitárias rigorosas no ambiente de produção para evitar que microrganismos psicrotróficos contaminem o leite (já que a maior parte deles tem origem no ambiente) e a redução no tempo de armazenamento a frio é sugerida para o controle de qualidade deste produto.

\section{Considerações finais}

Os microrganismos psicrotróficos que compõe a microbiota do leite cru refrigerado podem ser responsáveis pela produção de enzimas líticas e, consequentemente, pela degradação de componentes do leite. Para evitar perdas econômicas associadas à atividade enzimática, sugere-se que haja maior controle sanitário do ambiente de produção, assim como reduzidos períodos de armazenamento anteriores ao processamento térmico, visando reduzir a multiplicação de psicrotróficos proteolíticos.

\section{Referências bibliográficas}

Almuzara, M., Barberis, C., Traglia, G., Famiglietti, A., Ramirez, M. S., \& Vay, C. (2015). Evaluation of matrix-assisted laser desorption ionization-time-of-flight mass spectrometry for species identification of nonfermenting Gram-negative bacilli. Journal of Microbiological Methods, 112, 24-27.

Arcuri, E. F., Silva, P. D. L., Brito, M. A. V. P., Brito, J. R. F., Lange, C. C., \& Magalhães, M. M. A. (2008). Contagem, isolamento e caracterização de bactérias psicrotróficas contaminantes de leite cru refrigerado. Ciência Rural, 38, 2250-2255. https://doi.org/10.1590/S010384782008000800025.

Baur, C., Krewinkel, M., Kranz, B., von Neubeck, M., Wenning, M., Scherer, S., Stoeckel, M., Hinrichs, J., Stressler, T., \& Fischer, L. (2015). Quantification of the proteolytic and lipolytic activity of microorganisms isolated from raw milk. International Dairy Journal, 49, 23-29. https://doi.org/10.1016/j.idairyj.2015.04.005.

Borges, K. A., Reichert, S., Zanela, M. B., \& Fischer, V. (2009). Avaliação da qualidade do leite de propriedades da região do Vale do Taquari no estado do Rio Grande do Sul. Acta Scientiae Veterinariae, 37(1), 39-44. https://doi.org/10.22456/1679-9216.16185.

BRASIL Ministério da Agricultura, Pecuária e Abastecimento. Portaria n ${ }^{\circ} .101$ de 11 de agosto de 1993. Métodos analíticos oficiais para análise de produtos de origem animal e seus ingredientes II: métodos físicos e químicos. Diário Oficial da República Federativa do Brasil, Brasília, 1993.

BRASIL. Ministério da Agricultura, Pecuária e Abastecimento. Instrução normativa no 76, de 26 de novembro de 2018. Regulamentos Técnicos que fixam a identidade e as características de qualidade que devem apresentar o leite cru refrigerado, o leite pasteurizado e o leite pasteurizado tipo A. Diário Oficial da República Federativa do Brasil, Brasília, 30 de nov. de 2018.

Ismail, B., \& Nielsen, S. S. (2010). Invited review: Plasmin protease in milk: Current knowledge and relevance to dairy industry. Journal of Dairy Science, 93(11), 4999-5009. https://doi.org/10.3168/jds.2010-3122.

Jang, K.-S., \& Kim, Y. H. (2018). Rapid and robust MALDI-TOF MS techniques for microbial identification: a brief overview of their diverse applications. Journal of Microbiology, 56(4), 209216. https://doi.org/10.1007/s12275-018-7457-0.

Marchand, S., Duquenne, B., Heyndrickx, M., Coudijzer, K., \& Block, J. (2017). Destabilization and off-flavors generated by Pseudomonas proteases during or after UHT-processing of milk. International Journal of Food Contamination, 4(1), 1-7. https://doi.org/10.1186/s40550-016-0047-1.

Marchand, S., Vandriesche, G., Coorevits, A., Coudijzer, K., De Jonghe, V., Dewettinck, K., De Vos, P., Devreese, B., Heyndrickx, M., \& Block, J. (2009). Heterogeneity of heat-resistant proteases from milk Pseudomonas species. International Journal of Food Microbiology, 133(1-2), 68-77. https://doi.org/10.1016/j.ijfoodmicro.2009.04.027.

Moonga, H. B., Schoustra, S. E., van den Heuvel, J., Linnemann, A. R., Samad, M. S., Shindano, J., \& Smid, E. J. (2020). Composition and diversity of natural bacterial communities in mabisi, a traditionally fermented milk. Frontiers in Microbiology, 11, 1816. 
https://doi.org/10.3389/fmicb.2020.01816.

Mörschbächer, V., Rempel, C., \& Maciel, M. (2018). Microbiological quality of refrigerated raw milk in the dairy farm and after transport to the processing dairy plant. Arquivos Do Instituto Biológico, 84. https://doi.org/10.1590/1808-1657000422016.

Muir, D. D., \& Kjaerbye, H. (1996). Quality aspects of UHT cream. Federation Internationale de Laiterie-International Dairy Federation.

Nörnberg, M. F. B. L., Tondo, E. C., \& Brandelli, A. (2009). Bactérias psicrotróficas e atividade proteolítica no leite cru refrigerado. Acta Scientiae Veterinariae, 37(2), 157-163.

Pinto, C. L. O., Machado, S. G., Martins, M. L., \& Vanetti, M. C. D. (2015). Identificação de bactérias psicrotróficas proteolíticas isoladas de leite cru refrigerado e caracterização do seu potencial deteriorador. Revista Do Instituto de Laticínios Cândido Tostes, 70(2), 105-116. https://doi.org/10.14295/2238-6416.v70i2.401.

Reche, N. L. M., Thaler Neto, A., DOvideo, L., Felipus, N. C., Pereira, L. C., Cardozo, L. L., Lorenzetti, R. G., \& Picinin, L. C. A. (2015). Microbial multiplication in raw milk stored in direct expansion bulk tanks. Ciência Rural, 45(5), 828-834. https://doi.org/10.1590/0103-8478cr20140542.

Silveira, M. L. R., \& Bertagnolli, S. M. M. (2014). Avaliação da qualidade do leite cru comercializado informalmente em feiras livres no município de Santa Maria-RS. Vigilância Sanitária Em Debate: Sociedade, Ciência \& Tecnologia, 2(2), 75-80. https://doi.org/10.3395/vd.v2i2.135.

Stoeckel, M., Lidolt, M., Achberger, V., Glück, C., Krewinkel, M., Stressler, T., von Neubeck, M., Wenning, M., Scherer, S., \& Fischer, L. (2016a). Growth of Pseudomonas weihenstephanensis, Pseudomonas proteolytica and Pseudomonas sp. in raw milk: Impact of residual heat-stable enzyme activity on stability of UHT milk during shelf-life. International Dairy Journal, 59, 20-28. https://doi.org/10.1016/j.idairyj.2016.02.045.

Stoeckel, M., Lidolt, M., Stressler, T., Fischer, L., Wenning, M., \& Hinrichs, J. (2016b). Heat stability of indigenous milk plasmin and proteases from Pseudomonas: A challenge in the production of ultrahigh temperature milk products. International Dairy Journal, 61, 250-261. https://doi.org/10.1016/j.idairyj.2016.06.009.

Teh, K. H., Flint, S., Palmer, J., Andrewes, P., Bremer, P., \& Lindsay, D. (2014). Biofilm- An unrecognised source of spoilage enzymes in dairy products? International Dairy Journal, 34(1), 3240. https://doi.org/10.1016/j.idairyj.2013.07.002.

Vargas, D. P., Nörnberg, J. L., Mello, R. O., Sheibler, R. B., Milani, M. P., \& Mello, F. C. B. (2014). Correlações entre contagem bacteriana total e parâmetros de qualidade do leite. Revista Brasileira de Ciência Veterinária, 20(4), 241-247.

Vithanage, N. R., Dissanayake, M., Bolge, G., Palombo, E. A., Yeager, T. R., \& Datta, N. (2016). Biodiversity of culturable psychrotrophic microbiota in raw milk attributable to refrigeration conditions, seasonality and their spoilage potential. International Dairy Journal, 57, 80-90.

von Neubeck, M., Baur, C., Krewinkel, M., Stoeckel, M., Kranz, B., Stressler, T., Fischer, L., Hinrichs, J., Scherer, S., \& Wenning, M. (2015). Biodiversity of refrigerated raw milk microbiota and their enzymatic spoilage potential. International Journal of Food Microbiology, 211, 57-65. https://doi.org/10.1016/j.ijfoodmicro.2015.07.001.

Xin, L., Meng, Z., Zhang, L., Cui, Y., Han, X., \& Yi, H. (2017). The diversity and proteolytic properties of psychrotrophic bacteria in raw cows' milk from North China. International Dairy Journal, 66, 34-41. https://doi.org/10.1016/j.idairyj.2016.10.014.

Yuan, L., Sadiq, F. A., Burmølle, M., Wang, N. I., \& He, G. (2019). Insights into psychrotrophic bacteria in raw milk: a review. Journal of Food Protection, 82(7), 1148-1159. https://doi.org/10.4315/0362-028X.JFP-19-032.

Zhang, D., Palmer, J., Teh, K. H., \& Flint, S. (2020). Identification and selection of heat-stable protease and lipase-producing psychrotrophic bacteria from fresh and chilled raw milk during up to five days storage. LWT - Food Science and Technology, 134, 110165. https://doi.org/10.1016/j.lwt.2020.110165.

Histórico do artigo:

Recebido: 21 de setembro de 2021

Aprovado: 10 de outubro de 2021

Disponível online: 20 de dezembro de 2021
Licenciamento: Este artigoé publicado na modalidade Acesso Aberto sob a licença Creative Commons Atribuição 4.0 (CC-BY 4.0), a qual permite uso irrestrito, distribuição, reprodução em qualquer meio, desde que $\mathrm{o}$ autor $\mathrm{e}$ a fonte sejam devidamente creditados 